\title{
MicroRNA-125b is involved in atherosclerosis obliterans in vitro by targeting podocalyxin
}

\author{
XIAOBING LI, NA YAO, JUAN ZHANG and ZHENJIANG LIU
}

Department of Cardiology, The Second Xiangya Hospital of Central South University, Changsha, Hunan 410011, P.R. China

Received May 28, 2014; Accepted December 19, 2014

DOI: $10.3892 / \mathrm{mmr} .2015 .3384$

\begin{abstract}
Cardiovascular disease associated with oxidative stress, including atherosclerosis, is the leading cause of mortality worldwide. The accelerated proliferation and migration of vascular smooth muscle cells are the predominant characteristics of atherogenesis, and endothelial dysfunction is a major risk factor for the pathogenesis of atherosclerosis. Podocalyxin (PODXL), a type I member of the cluster of differentiation 34 family of sialomucins, functions as a pro-adhesive molecule. Emerging evidence has revealed the importance of micro (mi)RNAs in the cardiovascular system. The present study demonstrated that there was an inverse association between miRNA (miR)-125b and PODXL in human umbilical vein endothelial cells and human aortic vascular smooth muscle cells (HAVSMCs) treated with oxidized low-density lipoprotein (LDL) and platelet derived growth factor. Additionally, miR-125b had a suppressive function in cell proliferation and migration, at least partially via targeting PODXL in the HAVSMCs. Furthermore, the data suggested that the functions of miR-125b in arteriosclerosis obliterans may be associated with transgelin, lectin-type oxidized LDL receptor-1, vascular endothelial-cadherin, intercellular adhesion molecule-1, interleukin-6 and monocyte chemotactic protein-1. In conclusion, miR-125b was found to be important in arteriosclerosis obliterans by suppressing the expression of PODXL and may serve as a potential therepeutic target for the treatment of arteriosclerosis obliterans.
\end{abstract}

\section{Introduction}

Cardiovascular diseases associated with oxidative stress, including atherosclerosis, have become the leading cause of mortality worldwide (1). The accelerated proliferation and migration of vascular smooth muscle cells (VSMCs) are the

Correspondence to: Dr Zhenjiang Liu, Department of Cardiology, The Second Xiangya Hospital of Central South University, 139 Middle Renmin Road, Changsha, Hunan 410011, P.R. China E-mail: csuliuzhenjiang@163.com

Key words: atherosclerosis obliterans, podocalyxin, in vitro, microRNA-125b predominant characteristics of atherogenesis, and endothelial dysfunction is a major risk factor for the pathogenesis of atherosclerosis (2). Low-density-lipoprotein (LDL)-cholesterol is a clear predictor of cardiovascular disease and platelet-derived growth factor (PDGF), secreted by macrophages, exacerbates atherogenesis-stimulated VSMC proliferation and plaque neovascularisation (3). Podocalyxin (PODXL), a type I member of the cluster of differentiation 34 family of sialomucins, was originally observed in the epithelial cells of renal glomeruli (podocytes), which acted as a marker for the progression of kidney inflammatory diseases $(4,5)$. PODXL is also observed in vascular endothelium and in breast cancer, in which the expression levels appear to correlate with the metastatic capacity (6). A previous study reported that the overexpression of PODXL in Chinese hamster ovary cells increase their adhesion, migration and cellular interaction; indicating that PODXL acts as a pro-adhesive molecule (7). However, PODXL has also been reported to confer cell anti-adhesive properties in ovarian carcinoma (8). Despite this, it has been suggested that PODXL is associated with cell adhesion and migration $(9,10)$, indicating that it may be important in atherosclerosis.

Micro (mi)RNAs are 22 nucleotides long, non-coding RNA molecules, which can regulate gene expression either through translational inhibition or destabilization of target mRNA (11). Emerging evidence has revealed the importance of miRNAs in the cardiovascular system and a previous study demonstrated that let-7g miRNA downregulated the expression of the lectin-type oxidized LDL receptor-1 (Lox-1) resulting in the inhibition of vascular endothelial cell proliferation and migration (12). miRNA (miR)-21 has a pro-proliferative and anti-apoptotic effect on VSMCs and is involved in arteriosclerosis obliterans by targeting tropomyosin 1 (13). A previous study demonstrated, using an miRNA microarray analysis, that miR-125b was significantly downregulated in arteriosclerosis obliterans (14). However, its precise function in atherosclerosis obliterans remains to be elucidated.

The present study aimed to investigate the role of miR-125b in atherosclerosis obliterans and examine the association between miR-125b and PODXL.

\section{Materials and methods}

Cell culture and treatment. HUVECs and HAVSMCs were obtained from American Type Culture Collection (ATCC; Manassas, VA, USA) and cultivated in ATCC-formulated 
F-12K basic medium. The HUVECs were cultured in the complete growth medium supplemented with $10 \%$ fetal bovine serum (FBS; Gibco Life Technologies, Carlsbad, CA, USA), $0.1 \mathrm{mg} / \mathrm{ml}$ heparin (Sigma-Aldrich, St. Louis, MO, USA) and $0.04 \mathrm{mg} / \mathrm{ml}$ endothelial cell growth supplement (ECGS; Sigma-Aldrich). The HAVSMCs were cultured in complete growth medium supplemented with $10 \% \mathrm{FBS}, 0.05 \mathrm{mg} / \mathrm{ml}$ ascorbic acid (Sigma-Aldrich), $0.01 \mathrm{mg} / \mathrm{ml}$ insulin (Sigma-Aldrich), $0.01 \mathrm{mg} / \mathrm{ml}$ transferrin (Sigma-Aldrich), $10 \mathrm{ng} / \mathrm{ml}$ sodium selenite (Sigma-Aldrich), $0.03 \mathrm{mg} / \mathrm{ml}$ ECGS, $10 \mathrm{mM}$ HEPES (Sigma-Aldrich) and $10 \mathrm{mM}$ triethylsilane (Sigma-Aldrich). All the cells were cultured in $95 \%$ air and $5 \% \mathrm{CO}_{2}$ at $37^{\circ} \mathrm{C}$.

The HUVECs and HAVSMCs were treated with ox-LDL (75 $\mu \mathrm{g} / \mathrm{ml}$; Union Biotechnology, Hangzhou, China) and PDGF-BB (20 ng/ml; Union Biotechnology) for 24 or $48 \mathrm{~h}$ at $37^{\circ} \mathrm{C}$, to create a model of arteriosclerotic-like pathology. Ectopic expression of miR-125b in the cells was achieved by transfection with miR-125b mimics (GeneCopoeia, Inc. Rockville, MD, USA) using Lipofectamine 2000 (Invitrogen Life Technologies, Carlsbad, CA, USA). The pre- and anti-scramble controls were obtained from GeneCopoeia, Inc. The HUVECs and HAVSMCs were plated into six-well plates (Nest Biotechnology Co., Ltd., Shanghai, China) at a density of $1 \times 10^{5}$ cells $/ \mathrm{ml}$ and transfected for 24 or $48 \mathrm{~h}$. These transfected cells were used for total RNA or protein extraction and further analysis.

Reagents. Rabbit polyclonal antibodies against VE-cadherin (1:500 dilution; cat. no. B8251) and ICAM-1 (1:500 dilution; cat. no. B7113) were purchased from Assay Biotech Co., Inc. (Sunnyvale, CA, USA). Mouse monoclonal antibodies against PODXL (1:500 dilution; cat. no. BM0595), MCP-1 (1:1,000 dilution; cat. no. BM0186), IL-6 (1:1,000 dilution; cat. no. BM0009) and $\beta$-actin (1:2,000 dilution; cat. no. BM0272) were purchased from Abzoom Biolabs, Inc. (Dallas, TX, USA). Additional mouse monoclonal antibodies against SM-22 (1:1,000 dilution; cat. no. ab77442) and Lox-1 (1:1,500 dilution; cat. no. ab53202) were obtained from Abcam (Cambridge, MA, USA). Ox-LDL and PDGF-BB were obtained from Beijing Union Biotechnology (Beijing, China).

Lentiviral ( $L v$ ) transfection. The Lv-PODXL was purchased from GeneChem Co., Ltd. (Shanghai, China). The titer of the lentiviral recombinant vectors were $2 \times 10^{10}$ titer units (TU)/ml. The cells were plated and cultured in 6-well plates at a density of $5 \times 10^{5}$ cells $/ \mathrm{ml}$ at $37^{\circ} \mathrm{C}$, until cell fusion reached between 60 and $70 \%$. Subsequently, 2.5x10 4 TU/well Lv- PODXL lentivirus (conjugated to green fluorescent protein) were added to the cells at a multiplicity of infection of 50 for $48 \mathrm{~h}$. After $48 \mathrm{~h}$, the green fluorescence of the cells was observed using a AE31 microscope (MOTIC China Group Co., Ltd., Hong Kong) and the relative mRNA expression levels of PODXL were measured by reverse transcription quantitative polymerase chain reaction (RT-qPCR); the cells exhibiting $>90 \%$ fluorescence and a 15-fold increase in PODXL expression levels were considered to be sufficiently infected with the lentivirus. The infected cells were harvested for further analysis.
$R T$ - $q P C R$. The total RNA was extracted from the control cells, the cells treated with ox-LDL and the cells treated with PDGF-BB, using TRIzol reagent (Invitrogen Life Technologies), according to the manufacturer's instructions. The mRNA expression levels of PODXL, MCP-1 and IL-6 were detected using a SYBR green qPCR assay kit (Bio-Rad Laboratories, Inc., Hercules, CA, USA). The expression of $\beta$-actin was used as an endogenous control. The specific primers used were as follows: PODXL, forward 5'-TTTTACTCTTGCCCTCTC-3' and reverse 5'-CTTTCTTTCTGCCAAGAAAC-3' and $\beta$-actin, forward 5'-AGGGGCCGGACTCGTCATACT-3' and reverse 5'-GGCGGCACCACCATGTACCCT-3'. The relative expression of miR-125b was measured using an All-in-One ${ }^{\mathrm{TM}}$ miRNA qRT-PCR Detection kit (GeneCopoeia, Inc.). The specific primer sets for miRNA-103 and U6 and the qPCR mix were purchased from GeneCopoeia. The expression of U6 was used as an endogenous control. The PCR cycling conditions were set as follows: $95^{\circ} \mathrm{C}$ for $1 \mathrm{~min}$, followed by 35 cycles of $95^{\circ} \mathrm{C}$ for $15 \mathrm{sec}$ and $58^{\circ} \mathrm{C}$ for $15 \mathrm{sec}$. All experiments were performed in triplicate. Data were processed using the $2^{-\Delta \Delta C T}$ method (15).

Western blotting. The total cellular protein extracts $(50 \mu \mathrm{g})$ were prepared from the control cells, the cells treated with ox-LDL and the cells treated with PDGF-BB, separated on 10\% SDS-polyacrylamide gels (EMD Millipore, Billerica, MA, USA) and transferred onto nitrocellulose membranes (EMD Millipore). The membranes were treated with tris-buffered saline-Tween 20 (TBST; Auragene, Changsha, China) containing $50 \mathrm{~g} / \mathrm{ml}$ skimmed milk (BD Biosciences, Franklin Lakes, NJ, USA) overnight at $4^{\circ} \mathrm{C}$. The membranes were then incubated with antibodies targeting VE-cadherin, ICAM-1, PODXL, MCP-1, SM-22 and Lox-1 in the recommended dilutions overnight at $4^{\circ} \mathrm{C}$, and with an antibody targeting $\beta$-actin $(1: 2,000$ dilution) at room temperature for $2 \mathrm{~h}$. Following the incubation with primary antibodies, the membranes were washed with TBST four times. The membranes were then washed and incubated with horseradish peroxidase-conjugated goat anti-mouse (cat. no. SA001) and goat anti-rabbit (cat. no. SA009) secondary antibodies (1:3,000 dilutions; Auragene) at room temperature for $1 \mathrm{~h}$. The signals were visualized using enhanced chemiluminescence substrate (EMD Millipore). $\beta$-actin was used as a loading control.

3-(4,5-dimethylthiazol-2-yl)-2,5 diphenyl tetrazolium bromide (MTT) assay. An MTT assay was used to assess the cell growth ability of the cells transfected with pre-miR125b or pre-scramble, following PDGF-BB treatment. An MTT assay kit (Sigma-Aldrich) was used according to the manufacturer's instructions. Briefly, $5 \times 10^{3}$ cells were plated into each well of 96-well plates and cultured for 0, 1, 2 and 3 days at $37^{\circ} \mathrm{C}$. The cells were incubated with $20 \mu \mathrm{MTT}(5 \mathrm{mg} / \mathrm{ml})$ at $37^{\circ} \mathrm{C}$ for $4 \mathrm{~h}$ at the end of each time-point and, subsequently, the reaction was inhibited by adding $150 \mu \mathrm{l}$ dimethyl sulfoxide (Amresco LLC, Solon, OH, USA) for $10 \mathrm{~min}$ at room temperature. Formazan production was detected at $570 \mathrm{~nm}$ using an enzyme immunoassay analyzer (MK3; Thermo Fisher Scientific, Waltham, MA, USA). 
Transwell assay. The HAVSMCs $\left(1 \times 10^{6}\right.$ cells), trafnsfected with either pre-miR125b or pre-scramble, were treated with $20 \mathrm{ng} / \mathrm{ml}$ PDGF-BB for $48 \mathrm{~h}$ and starved in serum-free medium for $24 \mathrm{~h}$ at $37^{\circ} \mathrm{C}$, prior to being resuspended in serum-free medium $\left(5 \times 10^{4}\right.$ cells $)$. The cells were added to the upper chamber, while the lower chamber was filled with Dulbecco's modified Eagle's medium (GE Healthcare Life Technologies, Logan, UT, USA) containing 10\% FBS. The chambers were BioCoat Matrigel Invasion Chambers from BD Biosciences. Following incubation for $24 \mathrm{~h}$, the cells attached to the lower chamber were fixed and stained with crystal violet (BD Biosciences) for $20 \mathrm{~min}$. The redundant crystal violet was washed with water and dried in air. The crystal violet in the membrane was dissolved in $10 \%$ acetic acid and the optical density at $570 \mathrm{~nm}$ was detected using an enzyme immunoassay analyzer.

Dual luciferase reporter assay. A fragment of the 3' untranslated region (UTR) of PODXL, containing the putative miR-125b binding site, was amplified and recombined immediately into a psiCHECK-2 vector (Promega Corporation, Madison, WI, USA) downstream of the luciferase gene sequence. A psiCHECK-2 construct containing the 3'UTR of PODXL, with a mutant sequence of miR-125b, was synthesized. Wild-type (Wt)-PODXL and mutant (Mut)-PODXL primers were purchased from Promega Corporation. HUVECs and HAVSMCs were plated into 24-well plates 1 day prior to transfection, at a density of $2 \times 10^{4}$ cells/wellandeithertheWt-PODXL-3'UTR-psi-CHECK2 or Mut-PODXL-3'UTR-psi-CHECK2 were co-transfected with the miR-125b mimics, miR-125b inhibitor (GeneCopoeia, Inc.), pre-scramble and pre-scramble inhibitor, respectively, using Lipofectamine 2000 (Invitrogen Life Technologies). The cells were incubated for $48 \mathrm{~h}$ and the luciferase activity was detected using a dual-luciferase reporter assay system (Promega Corporation) and normalized to the activity of Renilla.

Statistical analysis. Statistical analyses were performed using independent samples t-tests to compare between two groups or the one-way analysis of variance to compare differences between more than two groups with SPSS 13.0 statistical software (SPSS, Inc., Chicago, IL, USA), depending on the experimental conditions. The data are expressed as the mean \pm standard deviation. Compared with the respective controls, $\mathrm{P}<0.05$ was considered to indicate a statistically significant difference.

\section{Results}

Expression levels of $m i R-125 b$ and PODXL in HUVECs and HAVSMCs treated with ox-LDL and PDGF-BB. LDL and PDGF are effective predictors of atherosclerosis. Following treatment with high concentrations of ox-LDL and PDGF-BB, certain characteristics of atherosclerosis were observed. To elucidate the association between the expression of miR-125b and PODXL in atherosclerosis, HUVECs and HAVSMCs were treated with ox-LDL $(75 \mu \mathrm{g} / \mathrm{ml})$ and PDGF-BB $(20 \mathrm{ng} / \mathrm{ml})$ to create an atherosclerotic-like pathological model. As shown in Fig. 1, a significant upregulation of the mRNA expression
A

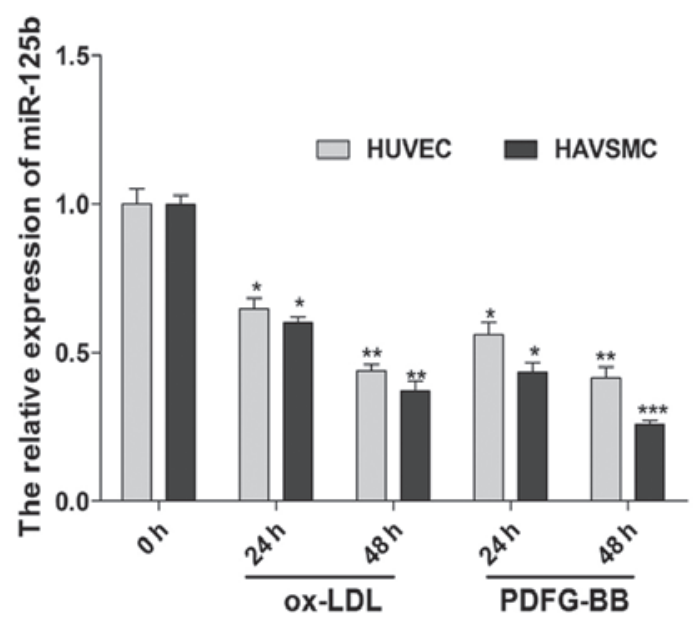

B

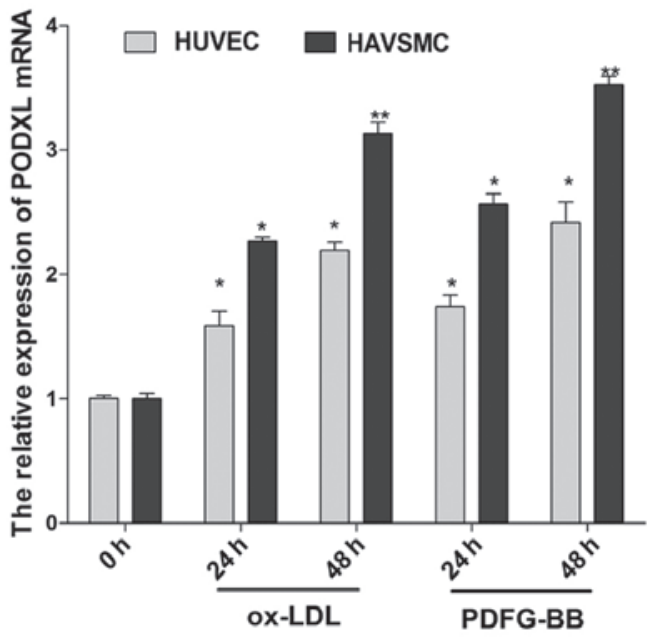

Figure 1. miRNA expression levels of PODXL and miR-125b in HUVECs and HAVSMCs following treatment with oxLDL and PDGF-BB for different durations. Reverse transcription quantitative polymerase chain reaction was performed to detect the expression of (A) miR-125b and (B) PODXL following treatment with oxLDL and PDGF-BB in the HUVECs and HAVSMCs. $\left({ }^{*} \mathrm{P}<0.05,{ }^{* * *} \mathrm{P}<0.01,{ }^{* * *} \mathrm{P}<0.001\right.$, vs. 0 h group). Data are expressed as the mean \pm standard deviation. HUVEC, human umbilical vein endothelial cell; HAVSMC, human aortic vascular smooth muscle cell; ox-LDL, oxidised low-density lipoprotein; PDFG, platelet-derived growth factor; PODXL, podocalyxin.

of PODXL was observed in the cells treated with ox-LDL and PDGF-BB, whereas the expression of miR-125b was significantly downregulated compared with that of the $0 \mathrm{~h}$ group. The results demonstrated that there was an inverse association between miR-125b and PODXL in the HUVECs and HAVSMCs with atherosclerotic-like pathological changes following treatment with ox-LDL and PDGF-BB.

miR-125b directly targets PODXL. According to an online miRNA target prediction database (TargetScan; www. targetscan.org), it was hypothesized that PODXL was a target of miR-125b (Fig. 2A). The present study revealed that there was an inverse association between the expression profiles of PODXL and miR-125b in the HUVECs and HAVSMCs treated 
A

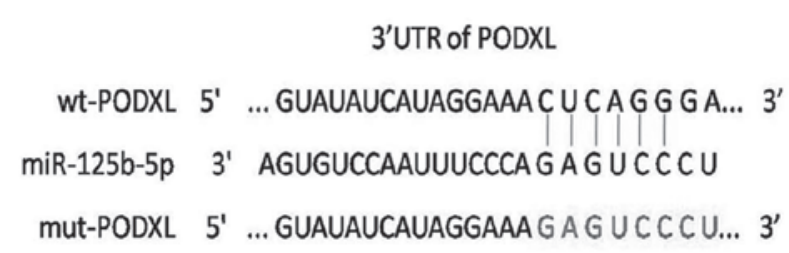

C

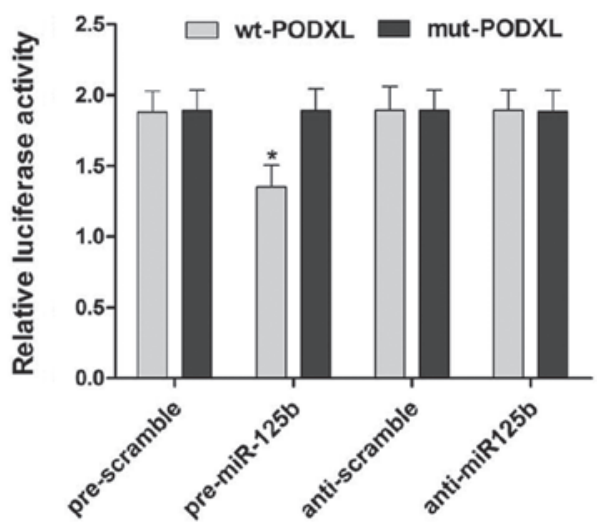

$\mathbf{E}$

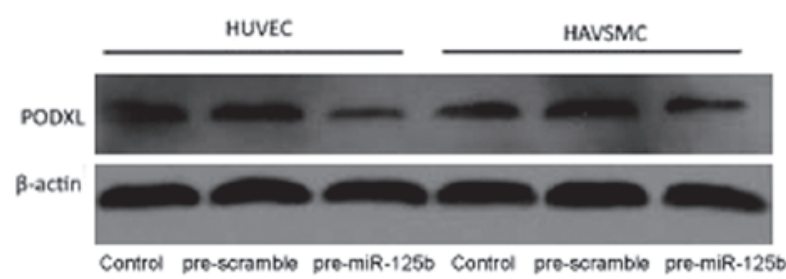

B

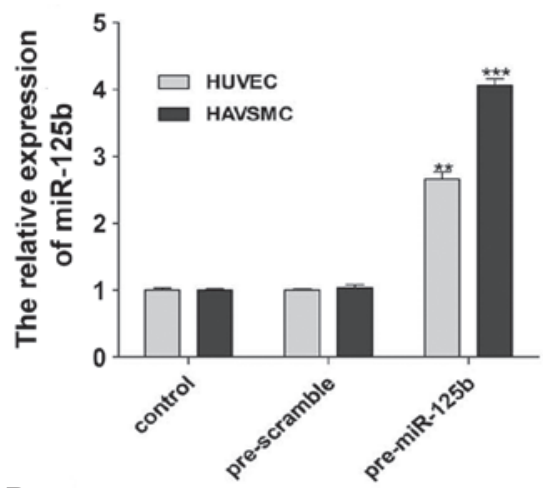

D

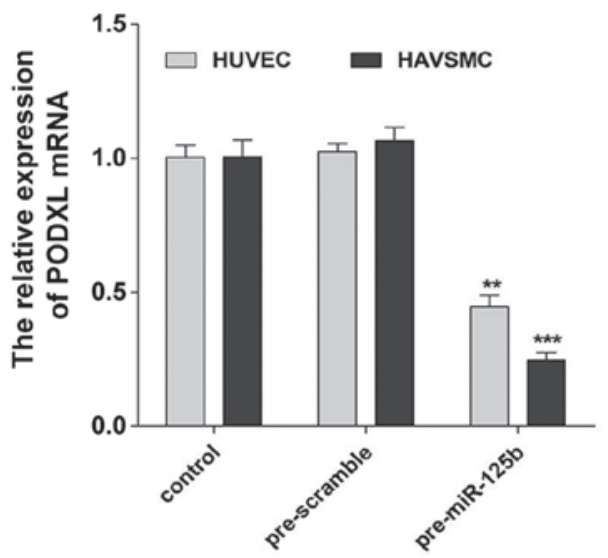

$\mathbf{F}$

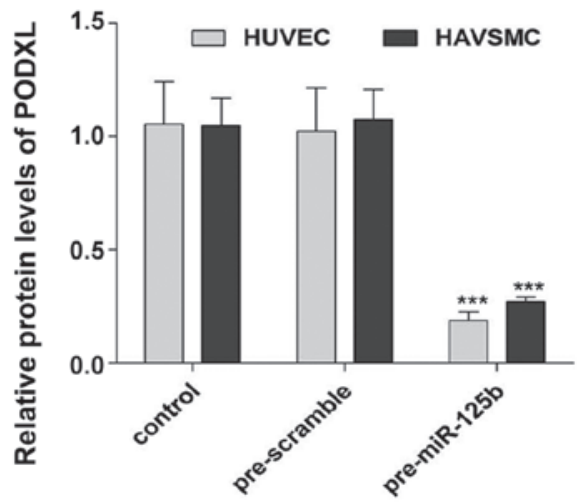

Figure 2. miR-125b directly targets PODXL. (A) Predicted miR-125b binding site within PODXL 3'UTR and its mutated version. (B) RT-qPCR was performed to detect the expression of miR-125b following transfection with pre-miR-125b and pre-scramble in the HUVECs and HAVSMCs. (C) Repression of luciferase activity by PODXL 3'UTR was dependent on miR-125b. Mutated PODXL 3'UTR abrogated the miR-125b mediated repression of luciferase activity. (D) RT-qPCR was performed to detect the mRNA expression of PODXL following transfection with pre-miR-125b and pre-scramble in the HUVECs and HAVSMCs. (E) Western blot analysis was performed to detect the protein expression of PODXL in the HUVECs and HAVSMCs treated with pre-miR-125b or pre-scramble. (F) Quantification of the protein expression of PODXL. $\left({ }^{*} \mathrm{P}<0.05,{ }^{* *} \mathrm{P}<0.01,{ }^{* * * *} \mathrm{P}<0.001\right.$, vs. control). Data are expressed as the mean \pm standard deviation. UTR, untranslated region; wt, wild-type; miR, microRNA; mut, mutant; PODXL, podocalyxin; HUVEC, human umbilical vein endothelial cell; HAVSMC, human aortic vascular smooth muscle cell; RT-qPCR, reverse transcription quantitative polymerase chain reaction.

with ox-LDL and PDGF-BB. To confirm whether PODXL was targeted by miR-125b, the HUVECs and HAVSMCs were transfected with miR-125b mimics or miR-scramble (Fig. 2B). The mRNA expression of PODXL was measured by RT-qPCR (Fig. 2D) and indicated the potential regulation of PODXL by miR-125b. Western blot analysis revealed that the upregulation of miR-125b significantly reduced the protein expression of PODXL compared with the cells transfected with the pre-scramble control (Fig. 2E and F). To further confirm whether the predicted binding site of miR-125b to the 3'UTR of PODXL was required for this regulation, the Wt-PODXL vector and miR-125b mimics or the scramble control were transfected into HUVECs and HAVSMCs. The relative luciferase activity of the miR-125b transfected cells was significantly repressed compared with the pre-scramble, anti-scramble and anti-miR-125b control groups (Fig. 2C). In 
A

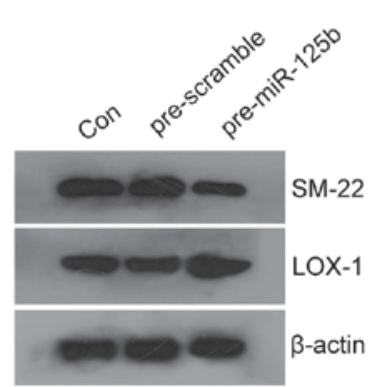

D

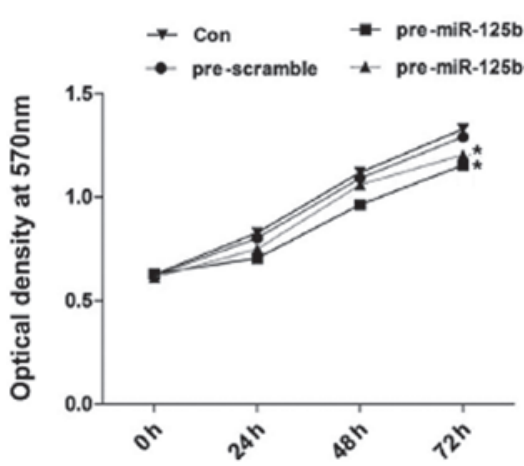

F

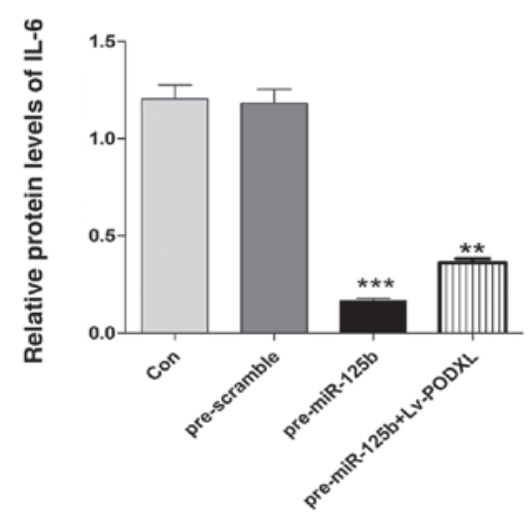

C

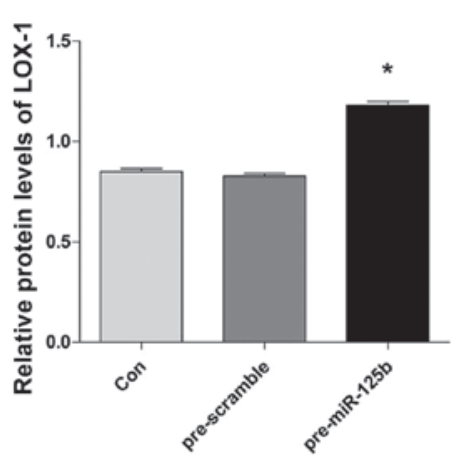

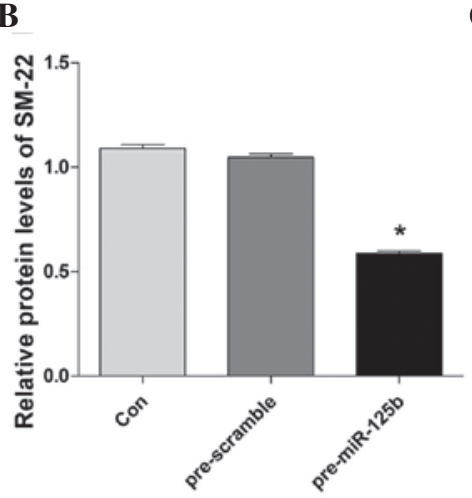

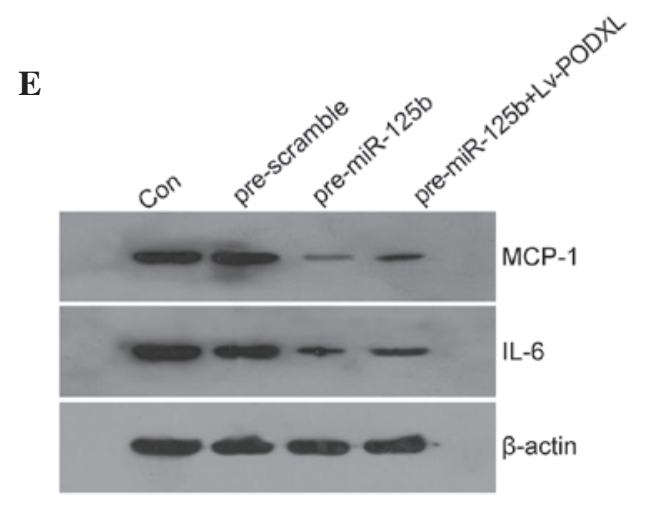

$\mathbf{G}$

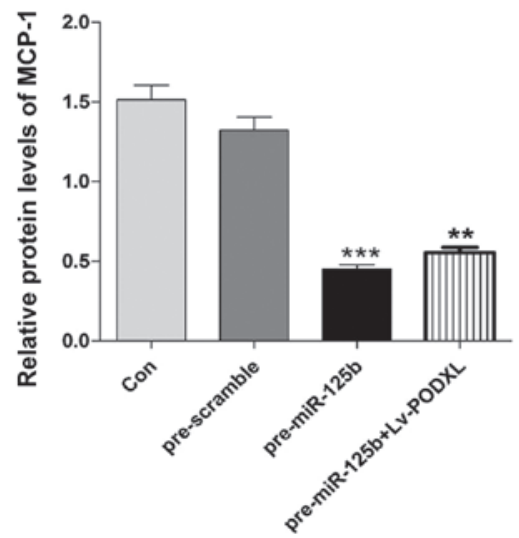

Figure 3. Effects of ectopic miR-125b on the expression levels of SM-22, Lox-1, IL-6 and MCP-1, and cell proliferation in the HAVSMCs treated with PDGF-BB (A) Western blotting was performed to detect the expression levels of (B) SM-22 and (C) Lox-1 and the band intensity was quantified. (D) Cell growth was evaluated using an MTT assay. The absorbance was measured at an optical density of $570 \mathrm{~nm}$. (E) Western blotting was performed to detect the expression levels of (F) IL-6 and (G) MCP-1 in HAVSMCs treated with PDGF-BB and the band intensity was quantified. ("P<0.05, ${ }^{* *} \mathrm{P}<0.01$ and ${ }^{* * * *} \mathrm{P}<0.001$, vs. control). The data are expressed as the mean \pm standard deviation. Con, control; miR, microRNA; Lox-1, lectin-type oxidized low-density lipoprotein receptor-1; MCP-1, monocyte chemotactic protein-1; IL-6, interleukin-6; HAVSMC, human aortic vascular smooth muscle cell; PDGF, platelet-derived growth factor; LV, lentivirus.

addition, the miR-125b-mediated repression of the luciferase activity was eliminated by the mutant putative binding site (Fig. 2C). These results confirmed that miR-125b directly targeted PODXL and regulated its expression at the transcriptional level.

Effect of miR-125b on the growth of HAVSMCs. To investigate whether miR-125b regulates the growth of HAVSMCs, cell growth was measured using an MTT assay following transfection with miR-125b mimics or scramble control. As shown in Fig. 3D, the HAVSMCs transfected with the miR-125b mimics exhibited significant inhibition of cell proliferation, compared with the control. However, when the HAVSMCs were co-transfected with pre-miR-125b and Lv-PODXL, the cell proliferation rate was moderately rescued. To elucidate the underlying mechanism of miR-125b in the HUVECs and HAVSMCs with atherosclerotic-like pathological changes, the protein expression levels of the inflammatory factors, IL-6 and MCP-1, were characterized in the HAVSMCs. The upregulation of miR-125b significantly decreased the protein 
A

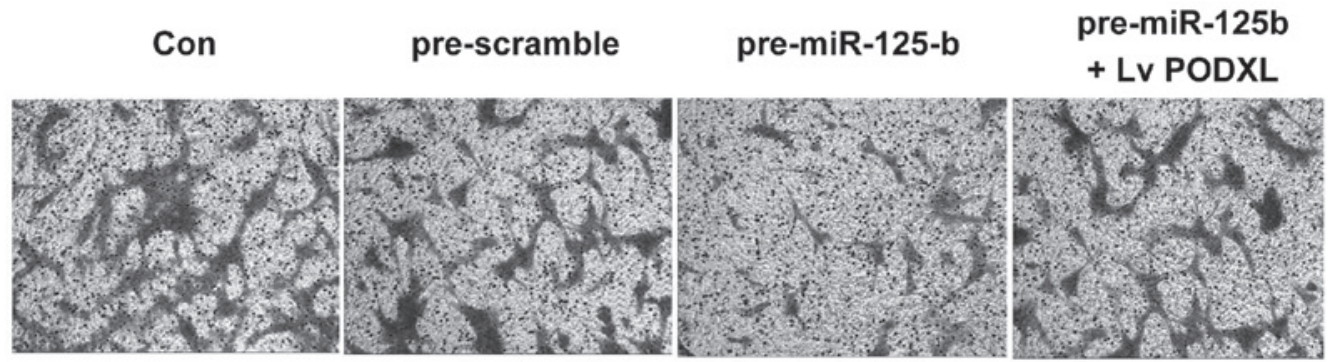

B

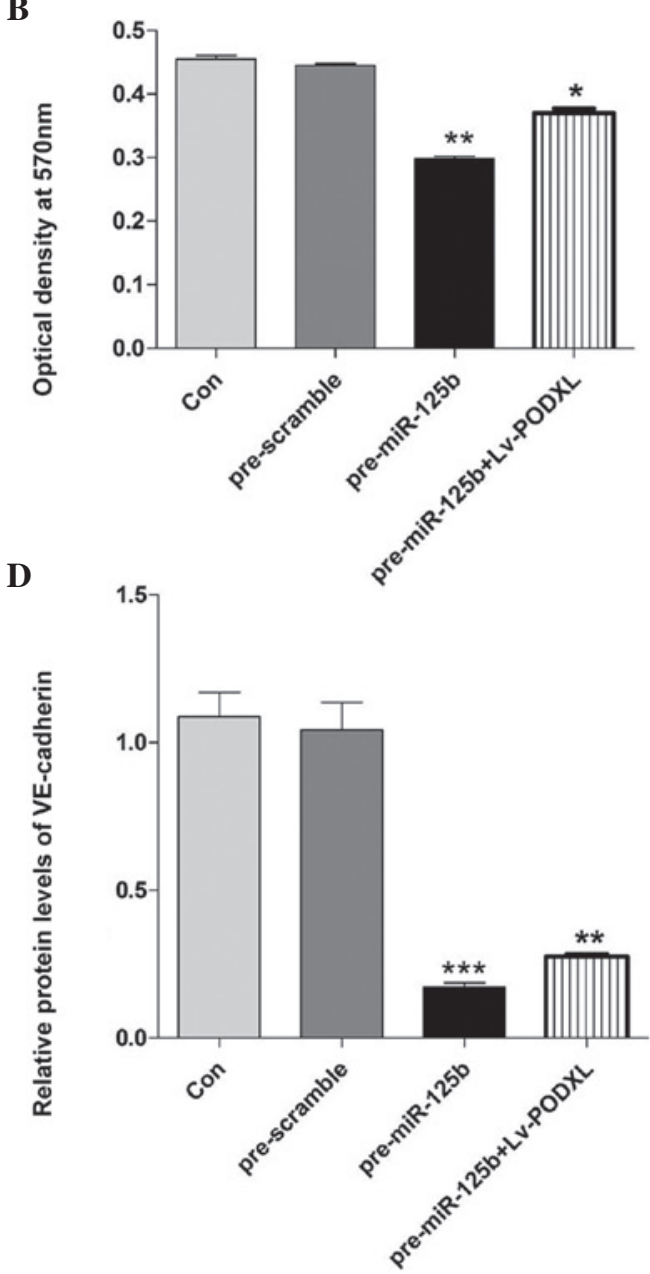

C

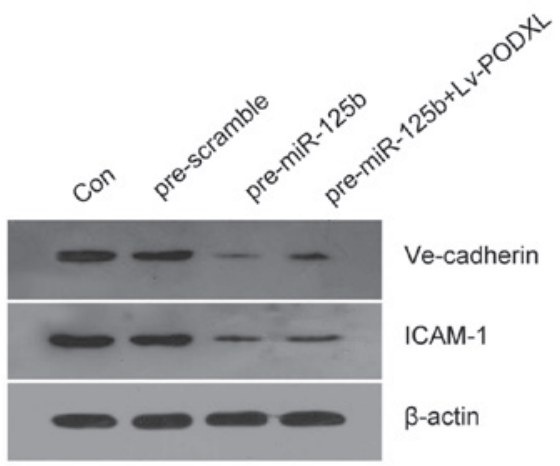

$\mathbf{E}$

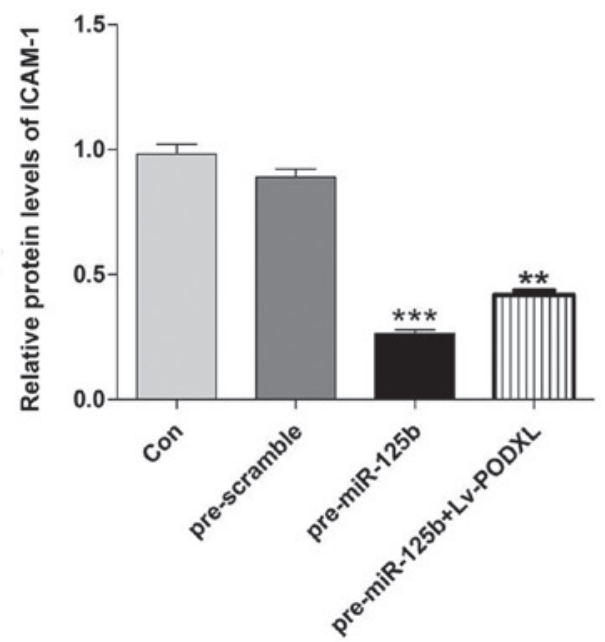

Figure 4. Effects of ectopic miR-125b on cell migration in HAVSMCs and the expression levels of VE-cadherin and ICAM-1 in HUVECs treated with PDGF-BB. (A) Representative images captured by a light microscope of migrated cells stained with crystal violet (magnification, x100). (B) Cell migration was determined using a Transwell assay and quantification. The absorbance was measured at an optical density of $570 \mathrm{~nm}$. (C) Western blotting was performed to detect the expression levels of (D) VE-cadherin and (E) ICAM-1 in the HUVECs treated with PDGF-BB and band intensity was quantified ("P $<0.05,{ }^{* *} \mathrm{P}<0.01$ and ${ }^{* * *} \mathrm{P}<0.001$, vs. control). The data are expressed as the mean \pm standard deviation. Con, control; miR, microRNA; VE, vascular endothelial; ICAM-1, intercellular adhesion molecule-1; HAVSMC, human aortic vascular smooth muscle cell; PDGF, platelet-derived growth factor; LV, lentivirus.

expression levels of IL-6 and MCP-1 (Fig. 3E-G) and this was reversed by the overexpression of PODXL (Fig. 4). The expression levels of SM-22 and Lox-1 were also detected by western blotting and, as shown in Fig. 3A-C, ectopic expression of miR-125b decreased the expression of SM-22 and increased the expression of Lox-1.

Transfection with miR-125b affects the migration of $H A V S M C s$ and affects the expression levels of VE-cadherin and ICAM-1 in HUVECs. To validate whether miR-125b regulates the migration of HAVSMCs, a Transwell assay was performed by transfecting either the miR-125b mimics or scramble control into HAVSMCs and treating with PDGF-BB. As shown in Fig. 4A, the HAVSMCs transfected with the miR-125b mimics exhibited significant inhibition of the PDGF-BB-induced migratory ability inhibition, compared with the pre-scramble control. However, when the HAVSMCs were co-transfected with pre-miR-125b and Lv-PODXL, the migratory ability was rescued. To elucidate the underlying mechanism, the protein expression levels of VE-cadherin and ICAM-1 were characterized in the HUVECs. Following transfection with the miR-125b mimics, it was found that 
the upregulation of miR-125b significantly decreased the expression levels of VE-cadherin and ICAM-1. However, the downregulation of VE-cadherin and ICAM-1, induced by miR-125b, was reversed by the overexpression of PODXL (Fig. 4).

\section{Discussion}

In the present study, upregulation of the mRNA expression of PODXL was observed in HUVECs and HAVSMCs treated with ox-LDL and PDGF-BB, which induced the overproliferation and migration of smooth muscle cells. PODXL, a cell surface glycoprotein, is closely associated with endoglycan and cell adhesion. It has been reported that PODXL increases the in vitro migration and invasion of breast and prostate cancer cells (16) and knock-down of PODXL significantly increases cell apoptosis in the presence of temozolamide in glioblastomas (17). In the endothelial venules, PODXL functions as an adhesive ligand for L-selectin-expressing leukocytes (18), indicating that PODXL has a pro-proliferative and pro-adhesive role. Results from the present study supported the hypothesis that PODXL acts as a pro-adhesive molecule.

Each miRNA can target the mRNA of several genes and can be involved in several physiological and pathophysiological processes. Emerging evidence suggests that mRNAs are important in vascular diseases through regulating the expression of target molecules in endothelial cells or smooth muscle cells. It was reported that the proliferation and migration of endothelial cells or smooth muscle cells was regulated by miR-424, miR-17-92 and miR-26a (19-21). Additionally, certain miRNAs are involved in ox-LDL- or PDGF-BB-induced atherosclerosis (22). Ox-LDL and PDGF-BB, effective predictors of cardiovascular disease, are important in the development of atherosclerosis by inducing overproliferation, monocyte adhesion and inflammation (23). The underlying mechanism and therapeutic strategies of atherosclerosis obliterans may be revealed by identification of the miRNAs involved and their targets. Although it has been confirmed that miR-125b is important in various types of cancer (24), few studies have investigated its functions in cardiovascular diseases, including atherosclerosis obliterans. Previous studies have demonstrated that miR-125b has a novel upstream role in the epigenetic regulation of inflammatory genes in VSMCs of diabetic mice, in that miR-125b targets the transcription factor, SP7, to regulate the transdifferentiation of VSMCs into osteoblast-like cells (14), and that is involved in the inhibition of osteoblastic differentiation by downregulating cell proliferation (25). The present study revealed that miR-125b was significantly downregulated in the HUVECs and HAVSMCs treated with ox-LDL or PDGF-BB, suggesting an association with overproliferation and migration of smooth muscle cells in the pathological process of atherosclerosis obliterans. To investigate the precise association and underlying mechanism between miR-125b and PODXL, the present study confirmed, using a luciferase reporter assay, that miR-125b directly targeted the PODXL gene through binding to a specific complementary site within its 3'UTR. Additionally, the results revealed that PODXL was effectively inhibited by the upregulation of the mRNA and protein expression of miR-125b in the HUVECs and HAVSMCs. Therefore, the decreased expression of miR-125b may account for the upregulation of PODXL in the pathological process of atherosclerosis obliterans.

The present study also demonstrated that the upregulation of miR-125b inhibited cell proliferation and migration in the HAVSMCs. However, cell proliferation was only moderately rescued by the overexpression of PODXL, suggesting that miR-125b had a suppressive function in cell proliferation, at least partially by targeting PODXL. Chronic inflammation in the arterial walls causes monocyte/macrophage recruitment and the migration and proliferation of VSMCs, promoting atherosclerosis obliterans (26). Upregulation of the inflammatory cytokines, IL-6 and MCP-1, has been associated with atherosclerosis obliterans (27-29). The dysregulation of miRNAs have been involved in several pathological processes of the cardiovascular diseases, including vascular atherosclerosis (30). Downregulation of miRNAs is associated with inflammation and is observed in patients with coronary artery disease (29). MicroRNAs, including miR-26a, miR-30b and miR-195, in the aortic valves of patients with aortic stenosis are decreased compared with those with aortic insufficiency (31). It was also reported that miR-125b is downregulated in calcified vessels in atherosclerotic mice and during vascular neointima formation (32). The present study revealed that the PDGF-BB-induced overexpression of miR-125b decreased the upregulation of IL-6 and MCP-1, which was consistent with a previous study suggesting that miR-125b was downregulated in response to lipopolysaccharide and tumor necrosis factor- $\alpha$ (33). In arteries, miR-125b is one of the most abundant microRNAs. This may explain why the overexpression of miR-125b significantly altered the gene expression of IL-6 and MCP-1, consistent with the hypothesis that small changes in miRNAs lead to widespread effects in cell function.

SM22- $\alpha$, a marker of cell differentiation, is associated with the transdifferentiation of ox-LDL-induced smooth muscle progenitor cell-derived smooth muscle-like cells into foam-like cells and, Lox-1 mediates this process (34). SM22- $\alpha$ has been used to identify vascular adventitial fibroblasts differentiating into myofibroblasts following vascular adventitial damage. Foam cell formation is a critical step in the development of atherosclerosis. In the present study, it was demonstrated that an increase in miR-125b induced the expression of Lox-1 and reduced the expression of SM-22 in HAVSMCs. It is possible that miR-125b regulates the expression of Lox-1 and SM-22 to inhibit the formation of foam cells.

Furthermore, the results revealed that the miR-125b mimic repressed the expression levels of VE-cadherin and ICAM-1, the master regulators of endothelial permeability and leukocyte transendothelial migration, in several vascular beds in the HUVECs. A previous study reported that ox-LDL induces a concentration-dependent upregulation of protein expression levels of MCP-1 and ICAM-1 genes, promoting the atherosclerosis obliterans process (35). The results from the present study demonstrated that miR-125b exhibited significant inhibition of the migratory ability of HAVSMCs, compared with the pre-scramble control. However, co-transfection with pre-miR-125b and Lv-PODXL, rescued the migratory ability of the HAVSMCs. These findings suggested that miR-125b inhibited the HAVSMCs from migrating into the blood vessel intima, most likely by repressing the expression of vascular cell adhesion molecules. 
In conclusion, the present study demonstrated for the first time, to the best of our knowledge, that miR-125b is involved in atherosclerosis obliterans, at least partially by targeting PODXL and signalling for the inflammatory cytokines and adhesion molecules involved in the pathological process of vascular atherosclerosis. These findings indicated that miR-125b is a potential small molecular target to prevent atherosclerosis obliterans. The underlying pathophysiological mechanisms of atherosclerosis remain to be elucidated and require further investigation.

\section{References}

1. Di Pietro M, Filardo S, De Santis F, Mastromarino P and Sessa R: Chlamydia pneumoniae and oxidative stress in cardiovascular disease: State of the art and prevention strategies. Int J Mol Sci 16: 724-735, 2014.

2. Endemann DH and Schiffrin EL: Endothelial dysfunction. J Am Soc Nephrol 15: 1983-1992, 2004.

3. Guo J, Li L, Wu YJ, et al: Inhibitory effects of Brazilin on the vascular smooth muscle cell proliferation and migration induced by PDGF-BB. Am J Chin Med 41: 1283-1296, 2013.

4. Michael AF, Blau E and Vernier RL: Glomerular polyanion. Alteration in aminonucleoside nephrosis. Lab Invest 23: 649-657, 1970.

5. Hara M, Yanagihara T, Takada T, et al: Podocalyxin on the glomerular epithelial cells is preserved well in various glomerular diseases. Nephron 67: 123-124, 1994.

6. Somasiri A, Nielsen JS, Makretsov N, et al: Overexpression of the anti-adhesin podocalyxin is an independent predictor of breast cancer progression. Cancer Res 64: 5068-5073, 2004.

7. Larrucea S, Butta N, Rodriguez RB, et al: Podocalyxin enhances the adherence of cells to platelets. Cell Mol Life Sci 64 2965-2974, 2007.

8. Cipollone JA, Graves ML, Köbel M, et al: The anti-adhesive mucin podocalyxin may help initiate the transperitoneal metastasis of high grade serous ovarian carcinoma. Clin Exp Metastasis 29: 239-252, 2012.

9. Fernández D, Horrillo A, Alquezar C, González-Manchón C, Parrilla R and Ayuso MS: Control of cell adhesion and migration by podocalyxin. Implication of Rac1 and $\mathrm{Cdc} 42$. Biochem Biophys Res Commun 432: 302-307, 2013.

10. Lin CW, Sun MS and Wu HC: Podocalyxin-like 1 is associated with tumor aggressiveness and metastatic gene expression in human oral squamous cell carcinoma. Int J Oncol 45: 710-718, 2014.

11. Huang BS, Luo QZ, Han Y, Li XB, Cao LJ and Wu LX: microRNA-223 promotes the growth and invasion of glioblastoma cells by targeting tumor suppressor PAX6. Oncol Rep 30: 2263-2269, 2013.

12. Chen KC, Hsieh IC, Hsi E, et al: Negative feedback regulation between microRNA let-7g and the oxLDL receptor LOX-1. J Cell Sci 124: 4115-4124, 2011.

13. Wang M, Li W, Chang GQ, et al: MicroRNA-21 regulates vascular smooth muscle cell function via targeting tropomyosin 1 in arteriosclerosis obliterans of lower extremities. Arterioscler Thromb Vasc Biol 31: 2044-2053, 2011.

14. Goettsch C, Rauner M, Pacyna N, Hempel U, Bornstein SR and Hofbauer LC: miR-125b regulates calcification of vascular smooth muscle cells. Am J Pathol 179: 1594-1600, 2011.

15. Arocho A, Chen B, Ladanyi M and Pan Q: Validation of the 2-DeltaDeltaCt calculation as an alternate method of data analysis for quantitative PCR of BCR-ABL P210 transcripts. Diagn Mol Pathol 15: 56-61, 2006.
16. Sizemore S, Cicek M, Sizemore N, et al: Podocalyxin increases the aggressive phenotype of breast and prostate cancer cells in vitro through its interaction with ezrin. Cancer Res 67: 6183-6191,2007.

17. Wu H, Yang L, Liao D, Chen Y, Wang W and Fang J: Podocalyxin regulates astrocytoma cell invasion and survival against temozolomide. Exp Ther Med 5: 1025-1029, 2013.

18. Sassetti C, Tangemann K, Singer MS, Kershaw DB and Rosen SD: Identification of podocalyxin-like protein as a high endothelial venule ligand for L-selectin: parallels to CD34. J Exp Med 187: 1965-1975, 1998.

19. Ghosh G, Subramanian IV, Adhikari N, et al: Hypoxia-induced microRNA-424 expression in human endothelial cells regulates HIF- $\alpha$ isoforms and promotes angiogenesis. J Clin Invest 120: 4141-4154, 2010

20. Kuhnert F and Kuo CJ: miR-17-92 angiogenesis micromanagement. Blood 115: 4631-4633, 2010.

21. Staszel T,Zapala B,Polus A, et al: Role of microRNAs in endothelial cell pathophysiology. Pol Arch Med Wewn 121: 361-366 2011.

22. $\mathrm{Wu} \mathrm{C}$, Gong $\mathrm{Y}$, Yuan J, et al: microRNA-181a represses ox-LDL-stimulated inflammatory response in dendritic cell by targeting c-Fos. J Lipid Res 53: 2355-2363, 2012.

23. Li D and Mehta JL: Antisense to LOX-1 inhibits oxidized LDL-mediated upregulation of monocyte chemoattractant protein-1 and monocyte adhesion to human coronary artery endothelial cells. Circulation 101: 2889-2895, 2000.

24. Zhou M, Liu Z, Zhao Y, et al: MicroRNA-125b confers the resistance of breast cancer cells to paclitaxel through suppression of pro-apoptotic Bcl-2 antagonist killer 1 (Bak1) expression. J Biol Chem 285: 21496-21507, 2010.

25. Mizuno Y, Yagi K, Tokuzawa Y, et al: miR-125b inhibits osteoblastic differentiation by down-regulation of cell proliferation. Biochem Biophys Res Commun 368: 267-272, 2008.

26. Williams KJ and Tabas I: Atherosclerosis and inflammation. Science 297: 521-522, 2002

27. Cushing SD, Berliner JA, Valente AJ, et al: Minimially modified low density lipoprotein induces monocyte chemotactic protein 1 in human endothelial cells and smooth muscle cells. Proc Natl Acad Sci USA 87: 5134-5138, 1990.

28. Rose CE Jr, Sung SS and Fu SM: Significant involvement of CCL2 (MCP-1) in inflammatory disorders of the lung. Microcirculation 10: 273-288, 2003.

29. Villeneuve LM, Kato M, Reddy MA, Wang M, Lanting L and Natarjan R: Enchanced levels of microRNA-125b in vascular smooth muscle cells of diabetic $\mathrm{db} / \mathrm{db}$ mice lead to increased inflammatory gene expression by targeting the histone methyltransferase Suv39h1. Diabetes 59: 2904-2915, 2010.

30. de Swiniarski R, Beatty D, Donoghue E, et al: Comparison of Schrodinger and Dirac coupled-channels analyses of the 28Si(p,p')28Si reaction at $500 \mathrm{MeV}$. Phys Rev C Nucl Phys 42: 1137-1140, 1990.

31. Nigam V, Sievers HH, Jensen BC, et al: Altered microRNAs in bicuspid aortic valve: a comparison between stenotic and insufficient valves. J Heart Valve Dis 19: 459-465, 2010.

32. Ji R, Cheng Y, Yue J, et al: MicroRNA expression signature and antisense-mediated depletion reveal an essential role of MicroRNA in vascular neointimal lesion formation. Circ Res 100: 1579-1588, 2007.

33. Tili E, Michaille JJ, Cimino A, et al: Modulation of miR-155 and miR-125b levels following lipopolysaccharide/TNF-a stimulation and their possible roles in regulating the response to endotoxin shock. J Immunol 179: 5082-5089, 2007.

34. Yu J, Li Y, Li M, Qu Z and Ruan Q: Oxidized low density lipoprotein-induced transdifferentiation of bone marrow-derived smooth muscle-like cells into foam-like cells in vitro. Int J Exp Pathol 91: 24-33, 2010.

35. Feng Y, Cai ZR, Tang Y, et al: TLR4/NF- $\kappa \mathrm{B}$ signaling pathway-mediated and oxLDL-induced up-regulation of LOX-1, MCP-1, and VCAM-1 expressions in human umbilical vein endothelial cells. Genet Mol Res 13: 680-695, 2014. 